\title{
Multimodaler Wirkansatz
}

_ Die muskelrelaxierenden und analgetischen Wirkmechanismen der 10\%igen Pfefferminzöllösung Euminz ${ }^{\oplus}$ sind in Studien belegt. So konnte nach der topi-

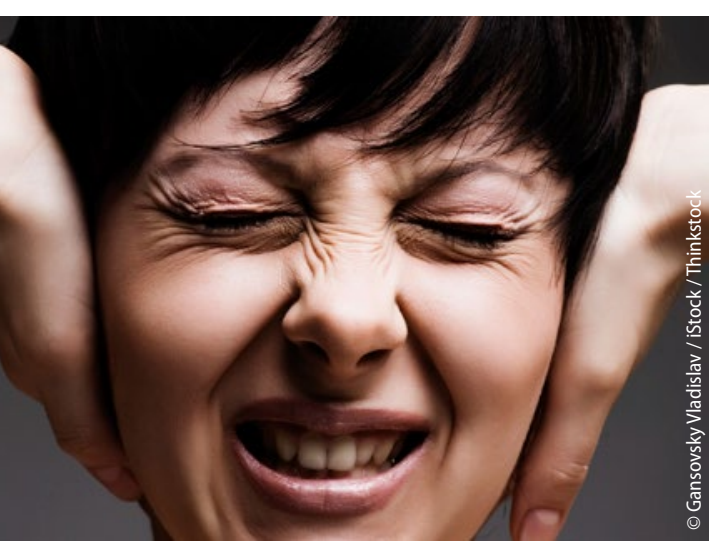

Quälende Kopfschmerzen sind zum Aus-der-Haut-Fahren! schen Applikation neben einer Schmerzhemmung, dem Abbau von Schmerzmediatoren sowie der Schmerzlinderung eine verbesserte Durchblutung der Hautkapillaren, eine Relaxation der perikranialen Muskulatur sowie eine Verbesserung der Befindlichkeit nachgewiesen werden. $10 \%$ iges Pfefferminzöl als lokal anzuwendendes Arzneimittel wird in mehreren Leitlinien zur Behandlung von leichten bis mittelschweren Kopfschmerzen vom Spannungstyp (ICD-10 G44.2) empfohlen. Für Erwachsene und Kinder ab sechs Jahren ist die großflächige Applikation von Euminz ${ }^{\circ}$ mittels des patentierten Dosier-Pads gut verträglich. Für Sechs- bis Zwölfährige ist das Präparat verordnungs- und erstattungsfähig.

Red.

- Nach Informationen von Klosterfrau

\section{Von-Basedow-Preis 2017}

Der von der Firma Henning unterstützte Von-Basedow-Preis 2017 der Deutschen Gesellschaft für Endokrinologie (DGE) wurde an Dr. Doreen Braun, Bonn, für ihre Arbeit zur Wirkung des chemischen Chaperons $\mathrm{Na}$ triumphenylbutyrat auf die mit dem AllanHerndon-Dudley-Syndrom (AHDS) assoziierte MCT8-Mutation verliehen.

Das AHDS ist ein X-chromosomales Syndrom, das mit einer schweren psychomotorischen Retardierung einhergeht. Ursache ist ein gestörter Transport des Schilddrüsenhormons T3, das zu einer bereits vorgeburtlich einsetzenden Unterversorgung des Gehirns mit Schilddrüsenhormon führt.

Bei einem Teil der Patienten sind die Transportproteine lediglich in ihrer Stabilität herabgesetzt. In diesen Fällen könnte den Patienten durch die Behandlung mit Natriumphenylbutyrat geholfen werden. Dabei handelt es sich um ein Medikament, das bereits zur Behandlung von Harnstoffzyklusstörungen zugelassen ist. Natriumphenylbutyrat ist im Zellmodel in der Lage, die Expression der Transportproteine zu steigern, womit eine erhöhte T3-Aufnahme in die Zelle verbunden ist. Der mögliche Therapieansatz soll nun in induzierten pluripotenten Stammzellen-Modellen weiter evaluiert werden.

\section{Dr. Silke Wedekind}

- Preisverleihung der Deutschen Gesellschaft für Endokrinologie, 60. Deutscher Kongress für Endokrinologie; Würzburg, März 2017

\section{Hochdosistherapie bei Vitamin-D-Mangel}

Im Januar 2017 hat InfectoPharm in Deutschland den Vertrieb von Colecalciferol bzw. Vi$\operatorname{tamin} D_{3}$ (HelioDrei ${ }^{\oplus}$ ) übernommen. Das Präparat ist zur Anfangsbehandlung von Vitamin-D-Mangelzuständen zugelassen und steht in zwei Wirkstärken (25.000 I.E. bzw. 100.000 I.E.) zur Verfügung. Es handelt sich um praktische Trinkampullen, deren Inhalt nach Orange schmeckt und mit einem Löffel Flüssigkeit eingenommen werden kann. Durch die gezielte Hochdosistherapie anstelle einer täglichen Gabe von niedrig dosiertem Vitamin $D_{3}$ kann die Compliance der Patienten verbessert werden. Bei nachgewiesenem Vitamin-D-Mangel ist das Arzneimittel voll erstattungsfähig.

Red.

- Nach Informationen von InfectoPharm 